Article

\title{
Characterization of Gut Microbiota in Prenatal Cold Stress Offspring Rats by 16S rRNA Sequencing
}

\author{
Jiasan Zheng, Tingting Zhu, Lipeng Wang, Jianfa Wang and Shuai Lian *(1) \\ College of Animal Science and Veterinary Medicine, Heilongjiang Bayi Agricultural University, \\ Daqing 163319, China; zjs3399@aliyun.com (J.Z.); ztt895731877@163.com (T.Z.); wlp921947@163.com (L.W.); \\ wjflw@sina.com (J.W.) \\ * Correspondence: lianshuai@byau.edu.cn
}

Received: 13 August 2020; Accepted: 31 August 2020; Published: 10 September 2020

Simple Summary: Prenatal stress, including prenatal cold stress has long-term effects on offspring's physical and mental health. Our previous study showed a reduction of anxiety-like behavior in offspring rats suffered from prenatal cold stress. It is well-known that gut microbiota was involved in a variety of physiological activities, such as emotion, cognition, and behavior. However, information on the comparison between prenatal cold stress and gut microbiota in offspring is limited. The current study compared the gut microbiota composition of the prenatal cold stress and non-stress offspring rats. Cold stressed during gestation period showed to change the offspring gut microbiota composition, and Bacteroides and Lactobacillus were significantly increased in prenatal cold stress offspring rat guts. With the hope, cold stress-induced negative effects of animals can be prevented by microbiological interventions.

\begin{abstract}
Our previous study showed a reduction of anxiety-like behavior in offspring rats suffered from prenatal cold stress; whether this was related to changes in the offspring gut microbiota is unclear. To obtain the evidence for the role of the gut microbiota in prenatal cold stress offspring, $16 \mathrm{~S}$ rRNA sequencing technology was used. Male and female offspring rat feces were collected from a room temperature group and a prenatal cold stress group $(n \geq 8)$ for microbial DNA extraction, followed by $16 \mathrm{~S}$ rRNA sequencing. The results indicated that prenatal cold stress could change the offspring's gut microbiota composition. Prenatal cold stress significantly upregulates Lactobacillus, Lactobacillus_gasseri, Bacteroides, and Bacteroides-acidifaciens in female offspring, whereas prenatal cold stress significantly reduced Lachnospiraceae and Prevotellaceae in male offspring. These data showed the characterization of gut microbiota in prenatal cold stress offspring rats, and these data suggest that microbiological intervention in the future can potentially prevent the negative effects caused by cold stress to animals.
\end{abstract}

Keywords: prenatal cold stress; offspring; $16 \mathrm{~S}$ rRNA; gut microbiota

\section{Introduction}

Many common and complex diseases could be traced back to the very beginning of life. The development of animals is a plastic process, from genotypic to phenotypic development, depending on the environment. The developing fetus responds to internal and external environmental conditions during the sensitive period of cell proliferation, differentiation, and maturation. These lead to changes in the functions of cells, tissues, and organs. In turn, these changes may have short-term or long-term consequences for health and disease susceptibility, either independently or through subsequent interactions between developmental processes and the environment [1-3].

Prenatal stress affects the hypothalamic-pituitary-adrenal axis (HPA) of offspring [4], the brain neurotransmitter system, sympathetic nervous system (SNS), and cognitive ability. It also alters the 
neuromodulation of immune function. Environmental factors may have a long-term effect on offspring physical and mental health [5], and nervous system; for example, prenatal cold stress, which can induce several diseases, causes offspring hypertension [6]. Prenatal stress caused brain development disorders was well established in rodents, associated with anxiety, depression, and other abnormal behaviors in offspring [7]. The offspring that experience prenatal psychosocial stress were at elevated risk of anxiety disorders [8]. Prenatal restraint stress caused long-term behavioral deficits in offspring through microbe and C-C motif chemokine ligand 2-dependent mechanisms [9].

Most of the above studies have confirmed that prenatal stress leads to increased anxiety-like behaviors in offspring. However, the results of the open-field test (OFT) and elevated plus-maze test (EPMT) in our previous studies showed that prenatal cold stress-induced the anxiety-like behavior of the offspring rats reduced [5,10]. It is now believed that gut microbiota was essential for early development and regulation of host physiology, central nervous system functions (such as cognitive function), and the neuroendocrine system (such as HPA axis) [11,12]. An increasing number of studies showed that stress could alter the gut microbiota [13,14], which in turn can influence behavior [15], and affect the biological and behavioral responses of the brain, including anxiety-like and depression-like behaviors. In addition, the gut microbiota is sensitive to temperature stress. Studies have shown that cold stress alters the gut microbiota in mice. Intestinal microorganisms transplanted from a cold exposed environment can increase sensitivity to insulin and increase intestinal size and absorption capacity [16].

Hence, we hypothesized that prenatal cold stress might regulate the behavior by altering the gut microbiota composition of the offspring. Because of the 16S rRNA gene sequencing could obtain the information on any microbial alterations present in the gut of animals, this study aims to find the gut microbiota characterization of prenatal cold stress diminished the anxiety-like behavior by 16S rRNA gene sequencing of the offspring fecal samples.

\section{Materials and Methods}

Procedures involving animals were approved by the Animal Care Committee of the Heilongjiang Bayi Agricultural University (Daqing, China). The experimental protocol was performed by the College of Animal Science and Veterinary Medicine, Heilongjiang Bayi Agricultural University (NO. BYAU20190213).

\subsection{Experimental Animals}

Twenty male $(280 \pm 20 \mathrm{~g})$ and thirty female $(230 \pm 20 \mathrm{~g}, 9-10$ weeks of age) SPF Wistar rats were purchased from Changsheng Co. Ltd. (Changchun, China).(the Wistar rats were sensitive to various nutrients and environment temperature, suitable for the study of various nutritional and stress) Management of animals feeding were referred to our previous studies $[5,10]$. The rats were kept in an artificial intelligence climate chamber for at least seven days to acclimate.

\subsection{Prenatal Stress}

A vaginal smear was taken to determine the proestrus of the female rats. On the day of proestrus, male and female rats mated in a cage in a ratio of 1 to 2 (one male and two female rats were placed in one cage). It was considered gestational day 0 when sperm was observed under the microscope (The males were removed from the cage when pregnancy was detected, and the specific operations are described in our previous studies [10,17]). On gestational day 14, the cold stress group of pregnant rats was placed in a $4{ }^{\circ} \mathrm{C}$ artificial intelligence climate chamber; the control group was continued to be kept at the temperature as $22 \pm 2{ }^{\circ} \mathrm{C}$. After parturition, the cold stressed groups were transferred to the room temperature climate chamber.

Twenty-one days after parturition, the offspring were divided into a cold female (CF), cold male (CM), room temperature female (RTF), and room temperature male (RTM) ( $n \geq 8$ in each group). The feces of each group were collected, frozen in liquid nitrogen to solidify (Solidify the bacteria in the sample so 
that the species and abundance of the sample do not change), then stored at $-80^{\circ} \mathrm{C}$ until the DNA was extracted for microbiome analysis. The timeline of the treatment protocol was shown in Figure 1.
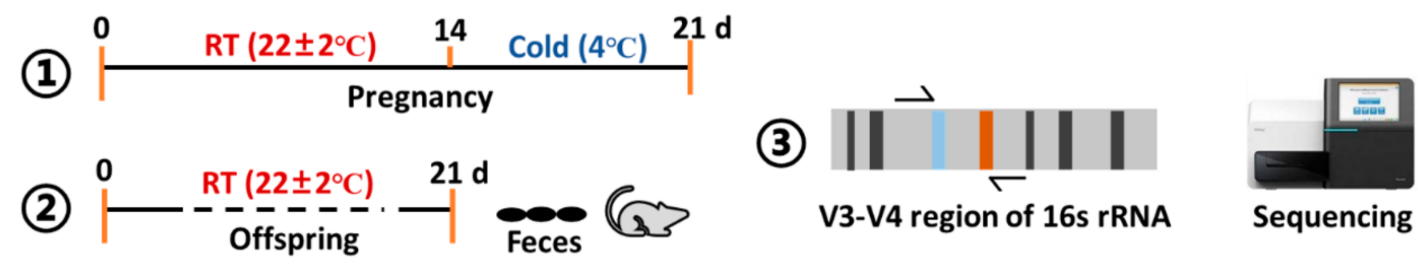

Figure 1. Treatment protocol and schematic workflow to examine the gut microbiota composition.

\subsection{Microbial Sequencing-16s rRNA}

DNA was extracted from feces using a QIA amp DNA Kit (Qiagen). After purity and concentration were measured, the DNA was diluted to $1 \mathrm{ng} / \mu \mathrm{L}$ with sterile water. The PCR was performed with diluted template DNA using region-specific primers (F:5'-CCTAYGGGRBGCASCAG-3' , R:5'-GGACTACH VGGGTWTCTAAT- $3^{\prime}$ ). PCR products were detected by electrophoresis, then purified with Gel Extraction Kit. Amplicon libraries were established using Ion Plus Fragment Library Kit. After Qubit quantification (Qubit dsDNA BR assay, Thermo Fisher, MA, USA) and library detection, the Ion S5TMXL (Thermo Fisher, MA, USA) was used for sequencing.

\subsection{Microbiome Data Processing}

Low-quality parts were sheared from reads by Cutadapt V1.91 (http://cutadapt.readthedocs.io/en/ stable/) first. The Raw Reads were obtained by preliminary quality control of truncated Barcode and the primer sequence. The sequences of Read were compared with the species annotation database to obtain Clean Reads (Table 1).

Table 1. Data preprocessing statistics and quality control information.

\begin{tabular}{cccccccc}
\hline Sample & Raw_Reads & Clean_Read & Base(nt) & AvgLen(nt) & Q20 & GC\% & Effective\% \\
\hline CF1 & 92,260 & 89,261 & $22,514,767$ & 252 & 82.14 & 52.13 & 96.75 \\
\hline CF2 & 83,650 & 80,149 & $20,243,525$ & 252 & 83.8 & 51.94 & 95.81 \\
\hline CF3 & 83,298 & 80,401 & $20,316,690$ & 252 & 82.54 & 52.07 & 96.52 \\
\hline CF4 & 83,297 & 80,125 & $20,214,254$ & 252 & 83.66 & 52.48 & 96.19 \\
\hline CF5 & 85,330 & 80,021 & $20,190,759$ & 252 & 83.37 & 51.75 & 93.78 \\
\hline CF6 & 85,139 & 80,086 & $20,229,884$ & 252 & 83.64 & 52.26 & 94.06 \\
\hline CF7 & 84,444 & 80,156 & $20,262,129$ & 252 & 82.6 & 52.91 & 94.92 \\
\hline CF8 & 93,369 & 90,450 & $22,854,929$ & 252 & 83.14 & 52.95 & 96.87 \\
\hline CF9 & 82,300 & 80,012 & $20,193,261$ & 252 & 83.36 & 49.78 & 97.22 \\
\hline CF10 & 83,407 & 80,210 & $20,227,906$ & 252 & 82.39 & 51.75 & 96.17 \\
\hline CF11 & 77,267 & 74,592 & $18,829,804$ & 252 & 88.38 & 51.82 & 96.54 \\
\hline CM1 & 85,000 & 80,135 & $20,229,046$ & 252 & 84.22 & 52.48 & 94.28 \\
\hline CM2 & 82,853 & 80,271 & $20,229,164$ & 252 & 83.8 & 50.55 & 96.88 \\
\hline CM3 & 85,402 & 80,218 & $20,248,437$ & 252 & 87.64 & 52.1 & 93.93 \\
\hline CM4 & 82,193 & 80,067 & $20,203,569$ & 252 & 89.1 & 53.63 & 97.41 \\
\hline CM5 & 85,686 & 80,027 & $20,196,156$ & 252 & 82.52 & 53 & 93.4 \\
\hline CM6 & 85,413 & 80,192 & $20,234,272$ & 252 & 81.6 & 51.65 & 93.89 \\
\hline
\end{tabular}


Table 1. Cont.

\begin{tabular}{|c|c|c|c|c|c|c|c|}
\hline Sample & Raw_Reads & Clean_Read & Base(nt) & AvgLen(nt) & Q20 & GC $\%$ & Effective $\%$ \\
\hline CM7 & 83,505 & 80,110 & $20,252,100$ & 252 & 80.29 & 51.82 & 95.93 \\
\hline CM8 & 82,851 & 80,161 & $20,257,659$ & 252 & 82.13 & 52.09 & 96.75 \\
\hline CM9 & 83,032 & 80,250 & $20,225,862$ & 252 & 85.33 & 50.51 & 96.65 \\
\hline CM10 & 94,549 & 91,345 & $23,051,906$ & 252 & 86.17 & 51.74 & 96.61 \\
\hline RTF1 & 89,793 & 85,797 & $21,722,030$ & 253 & 85.33 & 52.34 & 95.55 \\
\hline RTF2 & 84,096 & 80,166 & $20,241,950$ & 252 & 87.34 & 53.06 & 95.33 \\
\hline RTF3 & 85,131 & 80,209 & $20,322,631$ & 253 & 81.71 & 52.24 & 94.22 \\
\hline RTF4 & 82,078 & 80,181 & $20,237,808$ & 252 & 82.2 & 51.82 & 97.69 \\
\hline RTF5 & 103,541 & 99,559 & $25,148,508$ & 252 & 88.31 & 52.24 & 96.15 \\
\hline RTF6 & 85,361 & 80,235 & $20,254,767$ & 252 & 88.53 & 51.95 & 93.99 \\
\hline RTF7 & 71,314 & 68,814 & $17,387,481$ & 252 & 78.71 & 53.17 & 96.49 \\
\hline RTF8 & 84,943 & 80,114 & $20,195,893$ & 252 & 83.01 & 52.6 & 94.32 \\
\hline RTF9 & 84,557 & 80,148 & $20,248,562$ & 252 & 83.97 & 52.43 & 94.79 \\
\hline RTF10 & 82,732 & 80,286 & $20,269,324$ & 252 & 84.38 & 53.05 & 97.04 \\
\hline RTF11 & 83,449 & 80,090 & $20,217,831$ & 252 & 88.04 & 52.86 & 95.97 \\
\hline RTM2 & 83,144 & 80,084 & $20,225,142$ & 252 & 84.92 & 51.63 & 96.32 \\
\hline RTM3 & 83,169 & 80,105 & $20,284,526$ & 253 & 81.31 & 52.78 & 96.32 \\
\hline RTM4 & 83,560 & 80,200 & $20,254,101$ & 252 & 87.61 & 52.47 & 95.98 \\
\hline RTM5 & 85,973 & 79,380 & $20,034,819$ & 252 & 85.79 & 52.68 & 92.33 \\
\hline RTM6 & 83,612 & 80,295 & $20,278,888$ & 252 & 81.36 & 52.74 & 96.03 \\
\hline RTM7 & 82,830 & 80,147 & $20,222,220$ & 252 & 84.19 & 50.56 & 96.76 \\
\hline RTM8 & 84,339 & 80,215 & $20,236,766$ & 252 & 88.18 & 52.92 & 95.11 \\
\hline RTM9 & 83,915 & 80,047 & $20,206,904$ & 252 & 81.54 & 52.35 & 95.39 \\
\hline
\end{tabular}

To analyze the diversity of species composition of the samples, Uparse v7.0.1001 [18] was used for clustering for Clean Reads. The sequence cluster was called an Operational Taxonomic Unit (OTU) with $97 \%$ identity. Meanwhile, the representative sequences of OTU were selected. On the basis of its algorithmic theory, the sequence with the highest frequency in the OTUs were selected as the representative OTU sequence. Species annotation analysis was carried out by the Mothur method, and the SILVA SSUrRNA database [19] was used to obtain taxonomic information on each taxonomic level. Then, MUSCLE 3.8.31 software was used for multiple sequence alignment, and the system relations of all OTU representative sequences were obtained. Finally, the data of each sample were homogenized. Subsequent Alpha and Beta diversity analyses were based on homogenized data.

Alpha Diversity was used to analyze the microbial community diversity of the within-community. The dilution curve, rank abundance curve, and species accumulation curve were plotted using R software. The differences between groups of Alpha Diversity indices were also analyzed using the above software.

Beta Diversity was a comparative analysis of the microbial community composition of different samples. Principal Co-ordinates Analysis (PCoA) and Non-Metric Multi-Dimensional Scaling (NMDS) diagrams were drawn using R software. Differences in Beta diversity indices between groups were calculated by parametric and non-parametric tests using $R$ software. 


\subsection{Statistical Analysis}

The Alpha Diversity and Beta Diversity indices differences were analyzed by parametric and non-parametric tests. A $t$-test and Wilcoxon test were used for two groups. The Tukey test and Wilcoxon test were used for more than two groups. Correlation analysis was performed with the $\mathrm{R}$ software, $P<0.05$ were considered statistically significant.

\section{Results}

\subsection{OTU Cluster Abundance Analysis}

At the phylum level, Firmicutes, Bacteroidetes, Proteobacteria, Actinbacteria, Melainabacteria, Euryarchaeota, Tenericutes, Unidentified-Bacteria, Verrucomicrobia, and Chloroflexi were the top ten phyla in the offsprings' gut microbiota. The dominant phyla were Firmicutes, Bacteroidetes, and Proteobacteria, accounting for more than $97 \%$ of the phyla. The proportion of dominant phyla in each group is shown in Table 2 and Figure 2A,B.

Table 2. The proportion of dominant phylum in each group.

\begin{tabular}{cccc}
\hline Groups & Firmicutes & Bacteroidetes & Proteobacteria \\
\hline RYM & $57.02 \%$ & $38.04 \%$ & $3.18 \%$ \\
\hline CM & $47.57 \%$ & $47.28 \%$ & $2.48 \%$ \\
\hline RTF & $54.11 \%$ & $40.40 \%$ & $3.06 \%$ \\
\hline CF & $57.00 \%$ & $36.17 \%$ & $4.57 \%$ \\
\hline
\end{tabular}

\section{A}

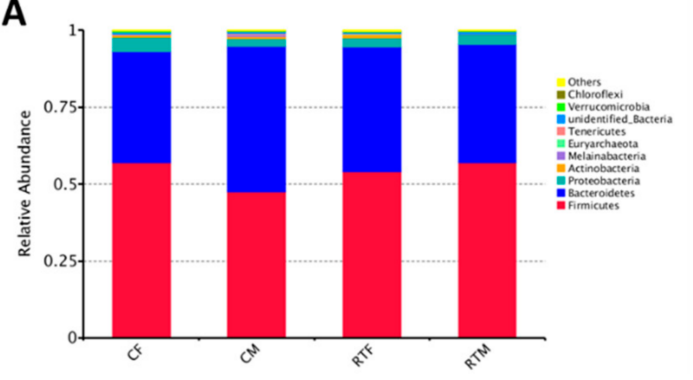

B

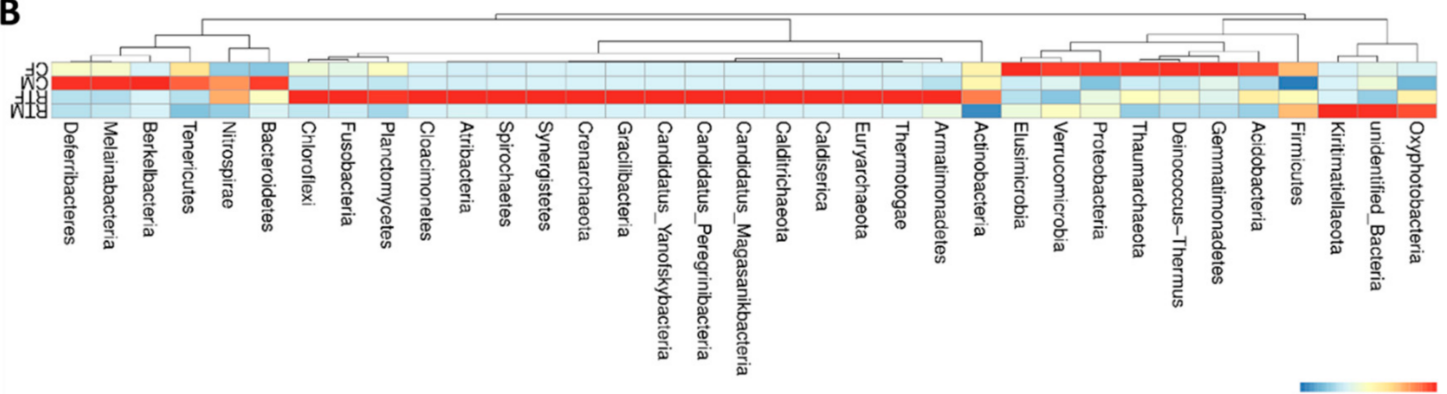

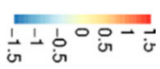

Figure 2. The proportion of dominant phyla in each group. (A) Relative abundance histogram of the gut microbiota of the offspring, (B) cluster heat map of the gut microbiota in the phylum level. Cold female (CF), cold male (CM), room temperature female (RTF), and room temperature male (RTM).

In the genus level, the top ten genera with the highest abundance were Bacteroides, Lactobacillus, Romboutsia, Unidentified-Lachnospiraceae, Blautia, Fusicatenibacter, Unidentified-Clostridiales, Lachnoclostridium, and Roseburia. The proportions in each group are shown in Table 3 and Figure 3A,B. 
Table 3. The top ten bacteria with the highest abundance at the genus level.

\begin{tabular}{ccccccccccc}
\hline Groups & Bacte & Lacto & Romb & Un.Lachn & Blaua & Fusica & Un.Clostri & Un.Entero & Lachn & Roseb \\
\hline RYM & 15.51 & 18.28 & 0.67 & 1.54 & 9.38 & 3.78 & 0.04 & 0.74 & 2.53 & 2.94 \\
\hline CM & 22.93 & 16.11 & 3.09 & 1.72 & 4.76 & 2.96 & 2.34 & 0.13 & 0.25 & 0.87 \\
\hline RTF & 9.03 & 14.39 & 8.20 & 5.84 & 5.52 & 3.24 & 0.12 & 0.08 & 0.41 & 0.76 \\
\hline CF & 20.97 & 28.75 & 4.99 & 1.52 & 2.40 & 0.31 & 0.59 & 1.21 & 0.51 & 0.37 \\
\hline
\end{tabular}

A

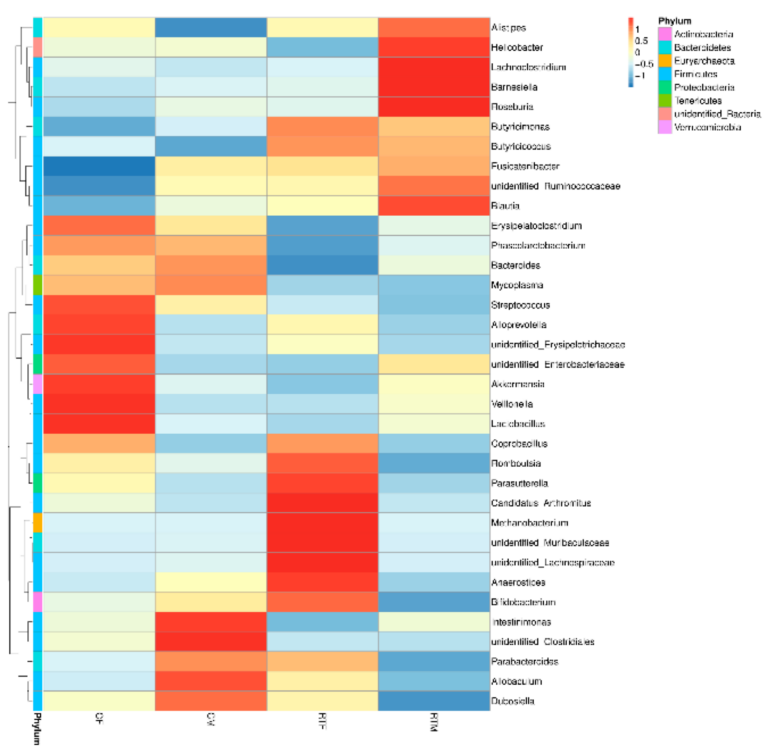

B

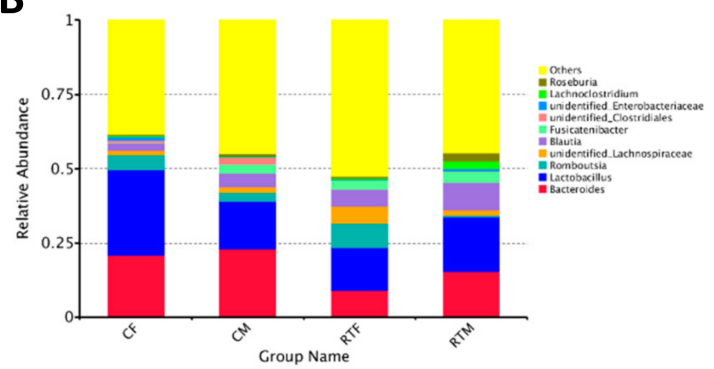

Figure 3. The top ten genera proportions in each group. (A) Cluster heat map of the gut microbiota at the genus level, (B) relative abundance histogram of the gut microbiota at the genus level. Cold female $(\mathrm{CF})$, cold male $(\mathrm{CM})$, room temperature female (RTF), and room temperature male (RTM).

\subsection{Alpha Diversity Analysis}

In order to verify whether these data fully reflected the species diversity of gut microbiota, we used the rarefaction curve to analyze each sample. The rarefaction curve and Shannon curve tended to be stable and straight as the sequencing deepened (Figure $4 \mathrm{~A}, \mathrm{~B}$ ). This indicated that the intestinal microbial diversity of the offspring had been fully detected. As shown in the species cumulative box plot (Figure 4C), with an increase in the number of test samples, the curves gradually became flat. This indicated that the number of samples in this experiment had reached the basic standard level of detection and sufficient to fully reflect the species richness.
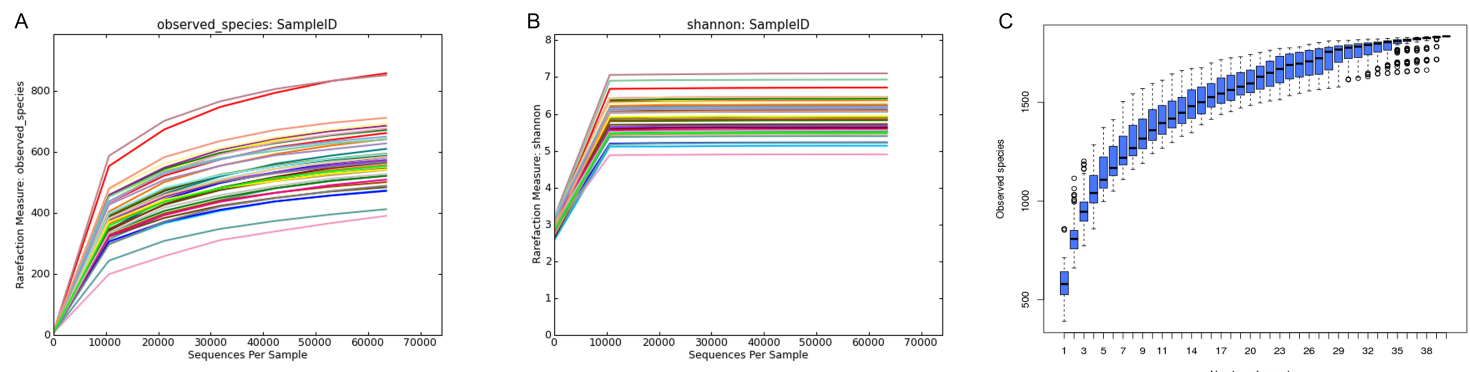

Figure 4. The rarefaction curve and Shannon curve. (A) Rarefaction curve of the offspring's gut microbiota, (B) Shannon curve of offspring gut microbiota, (C) species cumulative box plot of offspring gut microbiota. 


\subsection{Beta Diversity Analysis}

In order to analyze the influence of prenatal cold stress on the gut microbiota structure of offspring rats, we selected Non-Metric Multi-Dimensional Scaling (NMDS) and Principal Co-ordinates Analysis (PCoA) for intuitive analyses. As shown in Figure 5A, the confidence ellipses of group RTM and CM have been separated for the most part, and the confidence ellipses of RTF and CF have been completely separated; this indicated that the structure of gut microbiota in offspring rats had changed significantly, due to prenatal cold stress. However, the confidence ellipses of RTM and RTF offspring rats partially overlapped, indicating, thereby that the gut microbiota structure of offspring rats had sex differences. As shown in Figure 5B,C, the confidence ellipses of CM and CF have been completely separated; this also indicated that the influence of prenatal cold stress on the intestinal microflora of the offspring differed in male and female rats.
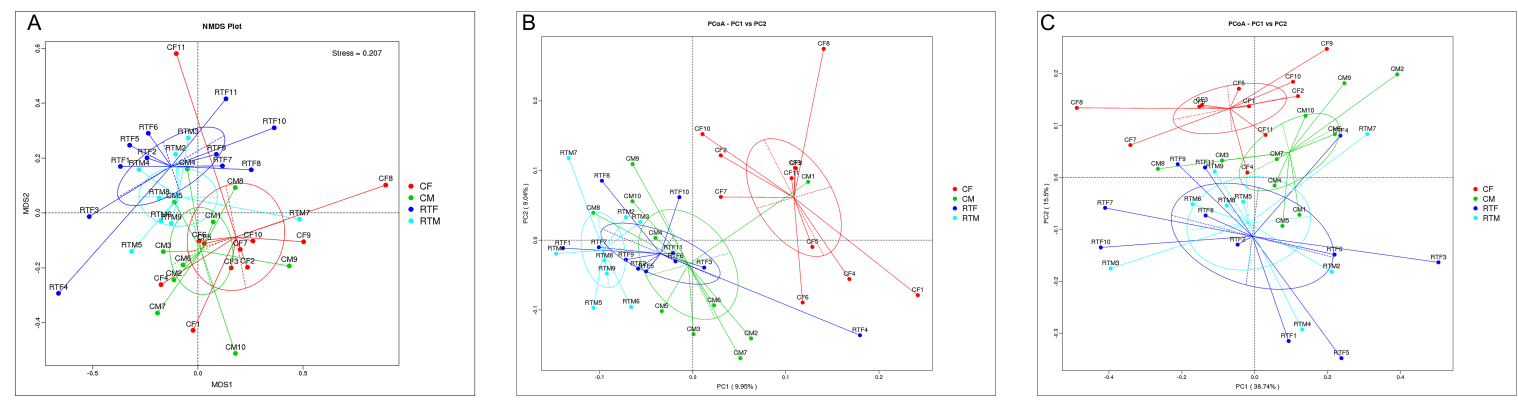

Figure 5. (A) Non-Metric Multi-Dimensional Scaling (NMDS) analysis of the gut microbiota of the offspring, (B) PcoA analysis of the gut microbiota of the offspring (unweighted), (C) Principal Co-ordinates Analysis (PcoA) analysis of the gut microbiota of the offspring (weighted). Cold female $(\mathrm{CF})$, cold male $(\mathrm{CM})$, room temperature female $(\mathrm{RTF})$, and room temperature male (RTM).

Multi Response Permutation Procedure (MRPP) was used to verify the prenatal cold stress effect on the gut microbiota structure of offspring rats. The results were consistent with the PCoA and NMDS analysis, $\mathrm{A}>0$ and $\mathrm{P}<0.05$ (Table 4). This indicated that prenatal cold stress had a significant effect on the gut microbiota structure of offspring rats.

Table 4. MRPP analysis.

\begin{tabular}{ccccc}
\hline Group & A & Observed-Delta & Expected-Delta & Significance \\
\hline CF-RTF & 0.05108 & 0.6782 & 0.7147 & 0.001 \\
RTF-RTM & 0.02732 & 0.683 & 0.7022 & 0.035 \\
CF-RTM & 0.04646 & 0.652 & 0.6837 & 0.001 \\
CM-RTF & 0.04115 & 0.6967 & 0.7266 & 0.004 \\
\hline
\end{tabular}

Cold female (CF), cold male (CM), room temperature female (RTF), room temperature male (RTM).

\subsection{Screening of Microorganisms in Response to Prenatal Cold Stress}

LDA Effect Size (LefSe) and $t$-test were used to identify the key microorganisms that showed the strongest response to prenatal cold stress. Compared to the RTM group, Lachnospiraceae and Prevotellaceae were significantly decreased in the CM group $(\mathrm{P}<0.05, \mathrm{LDA}=4)($ Figure 6A,B); compared to the RTF group, Bacilli, Lactobacillales, Lactobacillaceae, Lactobacillus, Bacteroides, Bacteroidaceae, and Lactobacillus_gasseri were significantly increased in the CF group $(\mathrm{P}<0.05, \mathrm{LDA}=4)$ (Figure 6C-E); compared to the RTM group, Lactobacillus_gasseri was significantly more in the RTF group (Figure 6F,G); compared to the CF group, Bacilli, Lactobacillales, Lactobacillaceae, and Lactobacillus were significantly increased in the CM group (Figure 6H,I). 
A

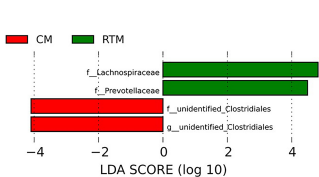

B
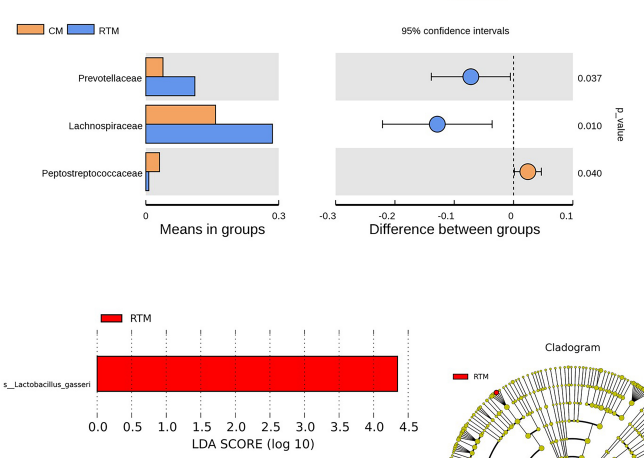

G

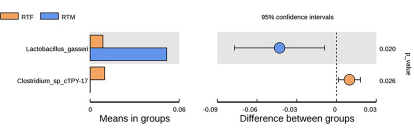

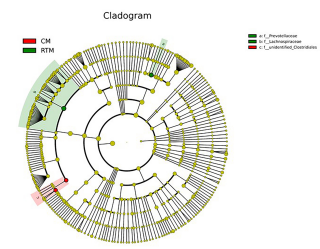

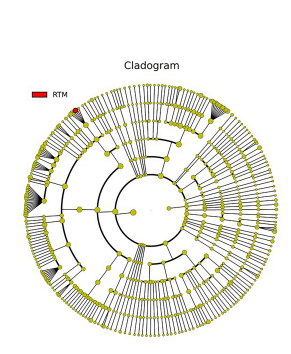

C
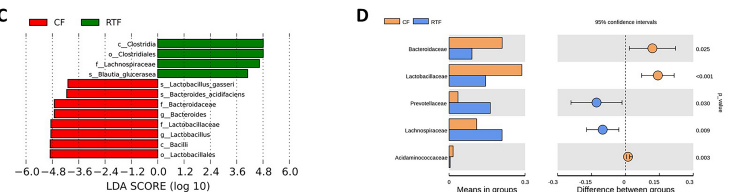

$\mathbf{E}_{\mathrm{R} \text { RI }}^{\mathrm{cF}}$
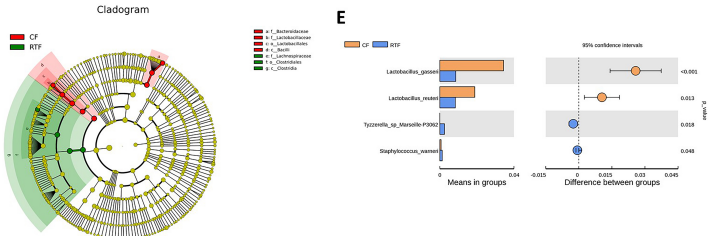

H

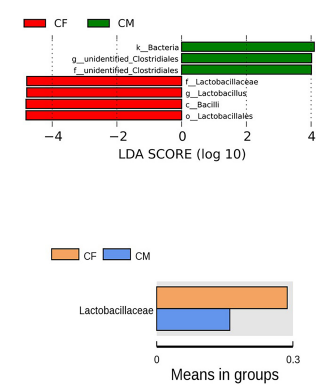

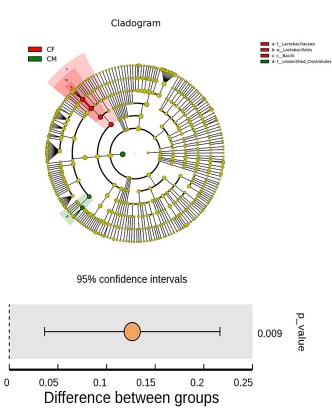

Figure 6. (A) LDA (Linear discriminant analysis) value distribution histogram between CM and RTM groups, (B) $t$-test analysis between CM-RTM groups, (C) LDA value distribution histogram between CF and RTF groups, (D) T-test analysis between CF-RTF groups (genus level), (E) $t$-test analysis between CF-RTF groups (species level), (F) LDA value distribution histogram between RTM and RTF groups, (G) $t$-test analysis between RTM-RTF groups, (H) LDA value distribution histogram between $\mathrm{CF}$ and CM groups, (I) $t$-test analysis between CF-CM groups. Cold female (CF), cold male (CM), room temperature female (RTF), and room temperature male (RTM).

\section{Discussion}

In our present study, the intestinal microbial diversity of the offspring had been fully detected, and alpha diversity analysis showed species richness of the offspring's gut microbiota. Beta diversity analysis showed the structure of gut microbiota had changed significantly, due to prenatal cold stress. We found that Bacteroides in the fecal samples of the CF group was more abundant than in the RTF group. One study demonstrated that the oral treatment of maternal immune activation (MIA) mouse offspring with human commensal Bacteroides fragilis can correct gut permeability, alter the microbial composition, and ameliorate defects in communicative, stereotypic, anxiety-like, and sensorimotor behaviors [20]. Prenatal cold stress may reduce the offspring's anxiety-like behavior by increasing the proportion of Bacteroidetes in the offspring's gut.

Moreover, we also found that prenatal cold stress caused an increase in the proportion of Lactobacillus in the offspring's gut. Lactobacillus is present in probiotics, which can regulate the response to stress and anxiety symptoms [21-23]. It has been demonstrated that feeding Lactobacillus rhamnosus increased GABA ( $\gamma$-aminobutyric acid) receptors in the hippocampus, and reduced stress-induced anxiety and depression-related behavior [24]. GABAergic neurotransmission in the hippocampus makes a decisive difference in the modulation of behavior and memory processes $[25,26]$. GABA-B receptors are related to mood and anxiety disorders, and blocking these receptors could reduce anxiety [27]. In agreement, in our previous study, we also found prenatal cold stress inhibited the expression of GABA-B2 receptors in the offspring rat's hippocampus. These results imply that the increase in Lactobacillus induced by prenatal cold stress, promotes the expression of GABA receptors in the hippocampus of the offspring, thereby reducing the offspring's anxiety-like behavior. In addition, 
the gut microbiota affects nervous system functions in the host [28,29]. Bacteroides and Lactobacillus produce large amounts of GABA [30,31]. Prenatal cold stress-induced an increase in Bacteroides and Lactobacillus in the offspring's gut; hence, we speculated that prenatal cold stress also increased GABA levels in the offspring.

In this study, we observed that the fecundity of Lactobacillus_gallinis in cold-stressed offspring was significantly higher than that in the room temperature group. It has been reported that Lactobacillus gallinis can produce a variety of bacteriocins, and can inhibit harmful bacteri. It is also involved in promoting intestinal stability and maintaining vaginal health, preventing allergic reactions, and inhibiting Helicobacter pylori. Prenatal cold stress-induced probiotic elevation may be regulated by the negative feedback mechanism of the organism.

Excitement and inhibition in healthy animals are two basic neural processes that are opposed to each other. A moderate amount of anxiety could help energize and mobilize individual energy to deal with stress, so increasing the chances of individual survival and continuation. When this anxiety was suppressed, the ability to respond to stress was also reduced. From our previous behavioral test results, prenatal cold stress could reduce the offspring's ability to react to stress. Moreover, the negative effects caused by cold stress to animals can be prevented by microbiological interventions.

\section{Conclusions}

In conclusion, Bacteroides and Lactobacillus were significantly increased in prenatal cold stress the offspring rats' gut. These data showed the characterization of gut microbiota in prenatal cold stress offspring rats, with the hope that cold stress-induced negative effects of animals can be prevented by microbiological interventions.

Author Contributions: Conceptualization, J.Z. and T.Z.; methodology, S.L.; software, L.W.; validation, S.L. and J.W.; formal analysis, L.W.; investigation, J.Z.; resources, S.L. and J.W.; data curation, J.Z. and T.Z.; writing-original draft preparation, J.Z. and L.W.; writing-review and editing, J.Z. and S.L.; visualization, L.W.; supervision, S.L.; project administration, S.L. and J.W.; funding acquisition, S.L. and J.W. All authors have read and agreed to the published version of the manuscript.

Funding: This research was funded by National Natural Science Foundation of China, grant number No. 31672513 and Heilongjiang Bayi Agricultural University Support Program for San Heng San Zong grant number No. TDJH201903 and No. ZRCQC201903.

Acknowledgments: This work was supported by Laboratory of Animal Stress Biology, Heilongjiang Bayi Agricultural University.

Conflicts of Interest: The authors declare no conflict of interest.

\section{References}

1. Gluckman, P.D.; Hanson, M.A. Living with the past: Evolution, development, and patterns of disease. Science 2004, 305, 1733-1736. [CrossRef]

2. Stoner, R.; Chow, M.L.; Boyle, M.P.; Sunkin, S.M.; Mouton, P.R.; Roy, S.; Wynshaw-Boris, A.; Colamarino, S.A.; Lein, E.S.; Courchesne, E. Patches of disorganization in the neocortex of children with autism. N. Engl. J. Med. 2014, 370, 1209-1219. [CrossRef]

3. Entringer, S.; Buss, C.; Kumsta, R.; Hellhammer, D.H.; Wadhwa, P.D.; Wüst, S. Prenatal psychosocial stress exposure is associated with subsequent working memory performance in young women. Behav. Neurosci. 2009, 123, 886-893. [CrossRef]

4. Glover, V.; O'Connor, T.G.; O'Donnell, K. Prenatal stress and the programming of the HPA axis. Neurosci. Biobehav. Rev. 2010, 35, 17-22. [CrossRef]

5. Lian, S.; Xu, B.; Wang, D.; Wang, L.; Li, W.; Yao, R.; Ji, H.; Wang, J.; Guo, J.; Li, S.; et al. Possible mechanisms of prenatal cold stress induced-anxiety-like behavior depression in offspring rats. Behav. Brain Res. 2019, 359, 304-311. [CrossRef]

6. Chen, K.; Sun, D.; Qu, S.; Chen, Y.; Wang, J.; Zhou, L.; Jose, P.A.; Yang, Y.; Zeng, C. Prenatal cold exposure causes hypertension in offspring by hyperactivity of the sympathetic nervous system. Clin. Sci. 2019, 133, 1097-1113. [CrossRef] 
7. Kubo, K.Y.; Kotachi, M.; Suzuki, A.; Iinuma, M.; Azuma, K. Chewing during prenatal stress prevents prenatal stress-induced suppression of neurogenesis, anxiety-like behavior and learning deficits in mouse offspring. Int. J. Med Sci. 2018, 15, 849-858. [CrossRef]

8. Beversdorf, D.Q.; Stevens, H.E.; Jones, K.L. Prenatal Stress, Maternal Immune Dysregulation, and Their Association with Autism Spectrum Disorders. Curr. Psychiatry Rep. 2018, 20, 76. [CrossRef]

9. Chen, H.J.; Antonson, A.M.; Rajasekera, T.A.; Patterson, J.M.; Bailey, M.T.; Gur, T.L. Prenatal stress causes intrauterine inflammation and serotonergic dysfunction, and long-term behavioral deficits through microbeand CCL2-dependent mechanisms. Transl. Psychiatry 2020, 10, 191. [CrossRef]

10. Lian, S.; Wang, D.; Xu, B.; Guo, W.; Wang, L.; Li, W.; Ji, H.; Wang, J.; Kong, F.; Zhen, L.; et al. Prenatal cold stress: Effect on maternal hippocampus and offspring behavior in rats. Behav. Brain Res. 2018, 346, 1-10. [CrossRef]

11. O'Mahony, S.M.; Clarke, G.; Borre, Y.E.; Dinan, T.G.; Cryan, J.F. Serotonin, tryptophan metabolism and the brain-gut-microbiome axis. Behav. Brain Res. 2015, 277, 32-48. [CrossRef]

12. Sherman, M.P.; Zaghouani, H.; Niklas, V. Gut microbiota, the immune system, and diet influence the neonatal gut-brain axis. Pediatric Res. 2015, 77, 127-135. [CrossRef]

13. Bailey, M.T.; Dowd, S.E.; Galley, J.D.; Hufnagle, A.R.; Allen, R.G.; Lyte, M. Exposure to a social stressor alters the structure of the intestinal microbiota: Implications for stressor-induced immunomodulation. Brain Behav. Immun. 2011, 25, 397-407. [CrossRef]

14. Bailey, M.T.; Dowd, S.E.; Parry, N.M.; Galley, J.D.; Schauer, D.B.; Lyte, M. Stressor exposure disrupts commensal microbial populations in the intestines and leads to increased colonization by Citrobacter rodentium. Infect. Immun. 2010, 78, 1509-1519. [CrossRef]

15. Kelly, J.R.; Borre, Y.; O’Brien, C.; Patterson, E.; El Aidy, S.; Deane, J.; Kennedy, P.J.; Beers, S.; Scott, K.; Moloney, G.; et al. Transferring the blues: Depression-Associated gut microbiota induces neurobehavioural changes in the rat. J. Psychiatr. Res. 2016, 82, 109-118. [CrossRef]

16. Chevalier, C.; Stojanović, O.; Colin, D.J.; Suarez-Zamorano, N.; Tarallo, V.; Veyrat-Durebex, C.; Rigo, D.; Fabbiano, S.; Stevanović, A.; Hagemann, S.; et al. Gut Microbiota Orchestrates Energy Homeostasis during Cold. Cell 2015, 163, 1360-1374. [CrossRef]

17. Lian, S.; Guo, J.; Wang, L.; Li, W.; Wang, J.; Ji, H.; Kong, F.; Xu, B.; Li, S.; Yang, H. Impact of prenatal cold stress on placental physiology, inflammatory response, and apoptosis in rats. Oncotarget 2017, 8, 115304-115314. [CrossRef]

18. Edgar, R.C. UPARSE: Highly accurate OTU sequences from microbial amplicon reads. Nat. Methods 2013, 10, 996-998. [CrossRef]

19. Quast, C.; Pruesse, E.; Yilmaz, P.; Gerken, J.; Schweer, T.; Yarza, P.; Peplies, J.; Glöckner, F.O. The SILVA ribosomal RNA gene database project: improved data processing and web-based tools. Nucleic Acids Res. 2013, 41, D590-D596. [CrossRef]

20. Hsiao, E.Y.; McBride, S.W.; Hsien, S.; Sharon, G.; Hyde, E.R.; McCue, T.; Codelli, J.A.; Chow, J.; Reisman, S.E.; Petrosino, J.F.; et al. Microbiota modulate behavioral and physiological abnormalities associated with neurodevelopmental disorders. Cell 2013, 155, 1451-1463. [CrossRef]

21. Rao, A.V.; Bested, A.C.; Beaulne, T.M.; Katzman, M.A.; Iorio, C.; Berardi, J.M.; Logan, A.C. A randomized, double-blind, placebo-controlled pilot study of a probiotic in emotional symptoms of chronic fatigue syndrome. Gut Pathog. 2009, 1, 6. [CrossRef]

22. Takada, M.; Nishida, K.; Kataoka-Kato, A.; Gondo, Y.; Ishikawa, H.; Suda, K.; Kawai, M.; Hoshi, R.; Watanabe, O.; Igarashi, T.; et al. Probiotic Lactobacillus casei strain Shirota relieves stress-associated symptoms by modulating the gut-brain interaction in human and animal models. Neurogastroenterol. Motil. Off. J. Eur. Gastrointest. Motil. Soc. 2016, 28, 1027-1036. [CrossRef]

23. Liao, J.F.; Hsu, C.C.; Chou, G.T.; Hsu, J.S.; Liong, M.T.; Tsai, Y.C. Lactobacillus paracasei PS23 reduced early-life stress abnormalities in maternal separation mouse model. Benef. Microbes 2019, 10, 425-436. [CrossRef]

24. Bravo, J.A.; Forsythe, P.; Chew, M.V.; Escaravage, E.; Savignac, H.M.; Dinan, T.G.; Bienenstock, J.; Cryan, J.F. Ingestion of Lactobacillus strain regulates emotional behavior and central GABA receptor expression in a mouse via the vagus nerve. Proc. Natl. Acad. Sci. USA 2011, 108, 16050-16055. [CrossRef] 
25. Crestani, F.; Lorez, M.; Baer, K.; Essrich, C.; Benke, D.; Laurent, J.P.; Belzung, C.; Fritschy, J.M.; Lüscher, B.; Mohler, H. Decreased GABAA-receptor clustering results in enhanced anxiety and a bias for threat cues. Nat. Neurosci. 1999, 2, 833-839. [CrossRef]

26. Dargaei, Z.; Bang, J.Y.; Mahadevan, V.; Khademullah, C.S.; Bedard, S.; Parfitt, G.M.; Kim, J.C.; Woodin, M.A. Restoring GABAergic inhibition rescues memory deficits in a Huntington's disease mouse model. Proc. Natl. Acad. Sci. USA 2018, 115, E1618-E1626. [CrossRef]

27. Ni, J.; Ohta, H.; Matsumoto, K.; Watanabe, H. Progressive cognitive impairment following chronic cerebral hypoperfusion induced by permanent occlusion of bilateral carotid arteries in rats. Brain Res. 1994, 653, 231-236. [CrossRef]

28. Fung, T.C.; Olson, C.A.; Hsiao, E.Y. Interactions between the microbiota, immune and nervous systems in health and disease. Nat. Neurosci. 2017, 20, 145-155. [CrossRef]

29. Cryan, J.F.; O’Riordan, K.J.; Cowan, C.S.M.; Sandhu, K.V.; Bastiaanssen, T.F.S.; Boehme, M.; Codagnone, M.G.; Cussotto, S.; Fulling, C.; Golubeva, A.V.; et al. The microbiota-gut-brain axis. Physiol. Rev. 2019, 99, 1877-2013. [CrossRef]

30. Strandwitz, P.; Kim, K.H.; Terekhova, D.; Liu, J.K.; Sharma, A.; Levering, J.; McDonald, D.; Dietrich, D.; Ramadhar, T.R.; Lekbua, A.; et al. GABA-Modulating bacteria of the human gut microbiota. Nat. Microbiol. 2019, 4, 396-403. [CrossRef]

31. Yunes, R.A.; Poluektova, E.U.; Dyachkova, M.S.; Klimina, K.M.; Kovtun, A.S.; Averina, O.V.; Orlova, V.S.; Danilenko, V.N. GABA production and structure of gadB/gadC genes in Lactobacillus and Bifidobacterium strains from human microbiota. Anaerobe 2016, 42, 197-204. [CrossRef]

(C) 2020 by the authors. Licensee MDPI, Basel, Switzerland. This article is an open access article distributed under the terms and conditions of the Creative Commons Attribution (CC BY) license (http://creativecommons.org/licenses/by/4.0/). 